\title{
ARTICLE \\ Assessing the allelotypic effect of two aminocyclopropane carboxylic acid synthase-encoding genes $M d A C S 1$ and MdACS3a on fruit ethylene production and softening in Malus
}

\author{
Laura Dougherty ${ }^{1}$, Yuandi Zhu ${ }^{1,2}$ and Kenong Xu
}

Phytohormone ethylene largely determines apple fruit shelf life and storability. Previous studies demonstrated that MdACS1 and MdACS3a, which encode 1-aminocyclopropane-1-carboxylic acid synthases (ACS), are crucial in apple fruit ethylene production. MdACS1 is well-known to be intimately involved in the climacteric ethylene burst in fruit ripening, while MdACS3a has been regarded a main regulator for ethylene production transition from system 1 (during fruit development) to system 2 (during fruit ripening). However, MdACS3a was also shown to have limited roles in initiating the ripening process lately. To better assess their roles, fruit ethylene production and softening were evaluated at five time points during a 20-day post-harvest period in $97 \mathrm{Malus}$ accessions and in 34 progeny from 2 controlled crosses. Allelotyping was accomplished using an existing marker (ACS1) for MdACS1 and two markers $\left(\mathrm{CAPS}_{866}\right.$ and $\mathrm{CAPS}_{870}$ ) developed here to specifically detect the two null alleles (ACS3a-G289V and Mdacs3a) of MdACS3a. In total, 952 Malus accessions were allelotyped with the three markers. The major findings included: The effect of MdACS1 was significant on fruit ethylene production and softening while that of MdACS3a was less detectable; allele MdACS1-2 was significantly associated with low ethylene and slow softening; under the same background of the MdACS1 allelotypes, null allele Mdacs3a (not ACS3a-G289V) could confer a significant delay of ethylene peak; alleles MdACS1-2 and Mdacs3a (excluding ACS3a-G289V) were highly enriched in $M$. domestica and $M$. hybrid when compared with those in $M$. sieversii. These findings are of practical implications in developing apples of low and delayed ethylene profiles by utilizing the beneficial alleles MdACS1-2 and Mdacs3a.

Horticulture Research (2016) 3, 16024; doi:10.1038/hortres.2016.24; Published online 18 May 2016

\section{INTRODUCTION}

To make fresh apple fruit available year-round for consumers, the controlled atmosphere (CA) storage technology has been adapted widely in the apple industry. The technology primarily employs low temperature, low $\mathrm{O}_{2}$ and high $\mathrm{CO}_{2}$ in combination with an ethylene production inhibitor 1-methylcyclopropene and others. Apple fruit can be stored for $>10$ months under optimal CA conditions. However, physiological disorders associated with CA storage, such as injuries induced by cold and $\mathrm{CO}_{2}$ and flesh browning induced by 1-methylcyclopropene, can cause substantial loss for storage operators. ${ }^{1-3}$ Such storage disorders have been reported for major apple varieties such as 'Empire' and 'Mclntosh' 2,4 and for rising cultivars such as 'Honeycrisp'.3 A strong need for new apples of long-shelf life and improved keeping quality with few or no storage disorders exists.

The gaseous phytohormone ethylene plays an important role in climacteric fruit ripening. The shelf life and storability of apple fruit are closely correlated with their ethylene production levels. Plant ethylene biosynthesis has been well-defined in Yang cycle that involves three enzymes: S-adenosylmethionine synthase, 1-aminocyclopropane-1-carboxylic acid (ACC) synthase (ACS) and ACC oxidase (ACO). ${ }^{5}$ The enzymes ACS and ACO have been the subject of extensive studies to better understand plant ethylene production. Studies in many plant species including tomato and apple have shown that ACS and ACO are encoded by gene families of multiple members, that is, the ACS family and the ACO family, respectively.

There are two systems of ethylene production in plants: system 1 occurs during plant/fruit growth and development; and system 2 is defined exclusively for the floral senescence and fruit-ripening stages. $^{6}$ In tomato, system 1 ethylene biosynthesis involves LeACS6, 1A and LeACO1, 3, 4; whereas system 2 uses LeACS2, 4 and ACO1, 4. ${ }^{7}$ In apple, at least five ACS (MdACS1-5) and four ACO (MdACO1-4) genes have been reported ${ }^{8,9}$ and these genes appear to be operating similarly in the two systems for ethylene production. MdACS1 is considered a system 2 gene; and its expression is highly correlated with the ethylene production burst in ripening apples. There are two alleles for the MdACS1 gene, MdACS1-1 and MdACS1-2, and the former is often associated with high ethylene production while the latter with lower ethylene production during fruit ripening. ${ }^{10-14}$ This observation has led to a marker-assisted selection strategy emphasizing on selection for allelotype (see Discussion for usage of term 'allelotype') MdACS1$2 / 2$ for long-shelf life apples. ${ }^{15}$ Indeed, some evidence suggests that modern apple-breeding practice has unintentionally favored selection for the MdACS1-2 allele in commercial apple cultivars, ${ }^{16}$ presumably for fruit of low ethylene and long-shelf life.

However, early-ripening cultivars showed faster fruit softening, regardless of their MdACS1 allelotypes. ${ }^{10}$ This is consistent with the observation that the polygalacturonase gene (MdPG1) involved in 
softening of fruit flesh is expressed irregularly among apple cultivars of identical MdACS1 allelotypes. ${ }^{12}$ Therefore, there are other factors also affecting fruit shelf life in addition to MdACS1. Interestingly, findings in a recent report have suggested that allele variations of another ACS gene (U73816), ${ }^{17}$ designated MdACS3a (AB243060), are an essential factor regulating apple fruit ripening and shelf life. ${ }^{18}$ There are two natural mutant alleles of the wildtype allele MdACS3a: One is the functional null allele MdACS3a$G 289 \mathrm{~V}$, arising from a point mutation that leads to an amino-acid substitution from $\mathrm{G}_{289}$ to $\mathrm{V}_{289}$ at an active region for the MdACS3A enzyme activity, resulting in a functionally inactive enzyme. In melon, a similar point mutation in a conserved active region of an ACS gene led to andromonoecy, a common sexual system in angiosperms characterized by carrying both male and bisexual flowers. ${ }^{19}$ This is an excellent example demonstrating that point mutations in conserved active regions of an ACS enzyme could confer a major phenotypic variation in plants. The other, a transcriptionally null allele Mdacs $3 a$, is characterized by non-detectable mRNA. Moreover, combinations of Mdacs3a and MdACS3a-G289V alleles, regardless of whether they are homozygous or heterozygous, are highly associated with lower ethylene production and long-shelf life. In the six apple varieties/selections of the two null alleles studied, all showed low ethylene production and long-shelf life, irrespective to their MdACS1 allelotypes and early, mid or late physiological maturation dates. ${ }^{18}$ Furthermore, the expression of MdACS3a is fruit tissue specific and detectable only during the transition from system 1 to 2 ethylene biosynthesis. $8,9,18$ These observations suggest that MdACS3a acts as a main regulator for the transition, and is thereby crucial in regulating the fruit-ripening process. ${ }^{18}$

In a more recent report, however, the allelotypes of MdACS3a were demonstrated to affect the ripening initiation of latematuring cultivars only, but not the early- or mid-maturing cultivars. $^{20}$ To better assess the roles of MdACS1 and MdACS3a, two approaches were taken in this study. The first approach was to estimate the allelotypic effect of the two genes by evaluating fruit ethylene production levels and softening rates in 97 diverse Malus accessions and 34 progeny from 2 controlled crosses. The second approach was to examine how variations in their allelotypic effect were associated with the frequency changes of the MdACS1 and MdACS3a alleles in M. domestica and M. hybrid as compared with those in $M$. sieversii, the major progenitor species of domestic apples, in 952 Malus accessions covering 53 Malus species. Allelotyping (see Discussion for usage of term 'allelotyping') of MdACS1 and MdACS3a was conducted using an existing marker for MdACS1 and two CAPS (cleaved amplified polymorphic sequence) markers specifically developed here to detect alleles ACS3a-G289V and Mdacs3a.

\section{MATERIALS AND METHODS}

\section{Plant materials}

Two sets of Malus accessions were used in this study, which have been planted and maintained in the Malus germplasm repository of the US Department of Agriculture (USDA) in Geneva, New York. The first set included a total of 952 accessions, covering 53 Malus species (Supplementary Table S1). Among them, Malus domestica of 508 accessions, $M$. hybrid (the breeding selections derived from crosses between $M$. domestica and other Malus species) of 146 and $M$. sieversii (the major progenitor species of $M$. domestica) of 78 were most commonly represented (Supplementary Table S1). The second set comprised 34 halfsib progeny selected from 2 interspecific crosses GMAL4592 ('Royal Gala' $\times$ PI613978) and GMAL4593 ('Royal Gala' $\times$ PI613981). 'Royal Gala', a widely grown apple cultivar ( $M$. domestica), has an allelotype MdACS1-2/2 and MdACS3a/MdACS3a-G289V for genes MdACS1 and MdACS3a, respectively. PI613978 and PI613981 are among the elite selections of M. sieversii collected from Kazakhstan, ${ }^{21}$ and they have the same allelotypes for the two ACS genes, that is, MdACS1-1/1 and MdACS3a/MdACS3a-G289V. Population GMAL4592 was used in one of our previous studies. ${ }^{22}$ Both
GMAL4592 and GMAL4593 were planted on their own seedling roots in 2004.

Measurements of fruit ethylene production and firmness

Fruit ethylene production and flesh firmness were measured for 97 of 952 Malus accessions in the first set and the 34 half-sib progeny in the second set as described previously. ${ }^{23}$ Briefly, for each accession, at least 25 fruits were harvested at a target maturity level as determined by the starch index of 4-6 according to the Cornell Starch Chart. ${ }^{24}$ The 25 fruits were evenly divided into 5 groups and were stored for $0,5,10,15$ and 20 days at room temperature $\left(20-25^{\circ} \mathrm{C}\right)$, respectively. Each fruit was weighed then enclosed in a gas-tight container (1.2 I) and kept for $1 \mathrm{~h}$ at room temperature. One milliliter of gas was sampled from the headspace in the container using a BD syringe (No. 309602, BD, Franklin Lakes, NJ, USA). The gas sample's ethylene concentration was measured with a gas chromatograph HP 5890 series II (Hewlett-Packard, Palo Alto, CA, USA) equipped with a flame ionization detector. Before the gas samples were assayed, the gas chromatograph was calibrated with standard ethylene gas (NO. 34489, Restek, Bellefonte, PA, USA) at a series of concentrations-0.01, 0.1, 0.5, 1, 5, 10 and 100 p.p.m.- to obtain the linear relation between ethylene peak area and concentration. The fruit ethylene production was calculated with the following formula:

$$
E=\left[C_{2} \mathrm{H}_{4}\right] \times\left(V_{1}-V_{2}\right) / W / T
$$

Where $E$ stands for fruit ethylene production rate in nanoliter per gram of fresh weight per hour $\left(n L ~ g^{-1} h^{-1}\right),\left[C_{2} H_{4}\right]$ for ethylene concentration in p.p. m., $V_{1}$ for the volume of container in $\mathrm{mL}, V_{2}$ for the volume of fruit in $\mathrm{mL}$ equivalent to fresh weight $(W)$ in grams and $T$ stands for the time in hours kept in the container.

Fruit flesh firmness was measured using a penetrometer (Fruit Tester, Wagner FTK100, Greenwich, CT, USA) with a probe of $11 \mathrm{~mm}$ in diameter. The probe tip was pressed vertically into the fruit pulp (after skin-disc removal) to a depth of $10 \mathrm{~mm}$. For larger fruits, four skin discs were removed from opposite sides of each fruit along the equator, and for smaller fruits, three skin discs were removed at roughly equal distance. The firmness readings were expressed in $\mathrm{kg} \mathrm{cm}^{-2}$, and firmness loss was measured by the percentage (\%) of firmness reduced at days 5 to 20 as compared with the firmness at day 0 . After the firmness was measured, fruits were sliced in half along the equator, dipped into a iodine-potassium iodide $\left(\mathrm{I}_{2}-\mathrm{KI}\right)$ solution, and then allowed the staining reaction for $>1 \mathrm{~min}$ before reading Cornell Starch Index. ${ }^{24}$

\section{Allelotyping of MdACS1 and MdACS3a}

Allelotyping of MdACS1 was conducted with marker ACS1 using primers ACS1-5F/R (Supplementary Table S2) as reported previously. ${ }^{10,15}$ However, allelotyping of MdACS3a was accomplished with two CAPS markers developed in this study using an online tool for identifying appropriate restriction enzymes ${ }^{25}$ (see Results). These two markers, named CAPS 866 and $\mathrm{CAPS}_{870}$, were capable of detecting the functional null allele MdACS3aG289V and the transcriptional null allele Mdacs $3 a$, respectively. In practice, the same primers ACS3a-289F/R (Supplementary Table S2) were used for PCR to amplify the targeted DNA fragment for both CAPS $_{866}$ and CAPS $_{870}$. PCRs were performed with 35 cycles of $94{ }^{\circ} \mathrm{C}$ for $30 \mathrm{~s}, 58^{\circ} \mathrm{C}$ for $30 \mathrm{~s}, 72^{\circ} \mathrm{C}$ for $1 \mathrm{~min}$, with an initial $94{ }^{\circ} \mathrm{C}$ for $5 \mathrm{~min}$ and a final extension of $72{ }^{\circ} \mathrm{C}$ for 10 min. Each PCR reaction mix was set in $10 \mu \mathrm{L}$ containing $20 \mathrm{ng}$ genomic DNA, 0.2 mm each dNTP, $0.5 \mu$ m of each primer, $2.5 \mathrm{~mm} \mathrm{MgCl}, 2 \mu \mathrm{L} 5 \times \mathrm{PCR}$ Colorless GoTaq Reaction Buffer and $1 \mathrm{U}$ of GoTaq DNA polymerase (Promega, Madison, WI, USA). To detect alleles MdACS3a-G289V and Mdacs $3 a$, the PCR products were restricted with enzymes BstNI and Taq ${ }^{a}$ I (New England Biolabs, Ipswich, MA, USA) following the manufacturer's instruction, respectively. The restricted PCR products were assayed by electrophoresis on $1.5 \%$ agarose gel and then stained with ethidium bromide for visualization and documentation as described previously. ${ }^{22}$

\section{Sanger DNA sequencing}

The PCR products amplified by primers ACS3a-289F/R (Supplementary Table S2) were directly sequenced using a DNA Sequencer ABI3730XL (Applied Biosystems, Foster City, CA, USA) at the Cornell University Biotechnology Resource Center (Ithaca, NY, USA). The reverse PCR primer ACS3a-289R was used for DNA sequencing. DNA sequence analyses were performed using software Sequencher 5.2 (Gene Codes Corporation, Ann Arbor, MI, USA). 
Statistical analysis

Pearson's correlation analysis and one-way analysis of variance (ANOVA) of ethylene production and fruit firmness were conducted with software JMP Pro 10.0 (SAS institute, Cary, NC, USA). Significance levels in comparison of the means were determined by $P<0.05$ (Student's $t$-test).

\section{RESULTS}

Evaluation of fruit ethylene production and softening

Fruit ethylene production and softening were evaluated in 97 of 952 Malus accessions (Supplementary Tables S1 and S3). Their mature date was determined by Cornell starch index, which had a mean $5.5 \pm 1.4$ at harvest. The 97 accessions varied widely not only in maturity date (from 16 August to 8 November 2011; Supplementary Figure S1a) and fruit weight (25.1-303.8 g, Supplementary Figure S1b), but also in ethylene production and firmness at harvest (day 0) and during the 20-day post-harvest period (Figures $1 \mathrm{a}$ and $\mathrm{b}$ ). At day 0 , for example, the ethylene levels ranged from $0.7 \mathrm{~nL} \mathrm{~g}^{-1}$ per $\mathrm{h}$ of PI588844 ('Fuji', M. domestica) to $679.3 \mathrm{~nL} \mathrm{~g}^{-1}$ per $\mathrm{h}$ of PI619168 (an accession of $M$. sylvestris), and fruit firmness varied from $3.8 \mathrm{~kg} \mathrm{~cm}^{-2}$ of PI589572 (E14-32, M. hybrid) to $12.7 \mathrm{~kg} \mathrm{~cm}^{-2}$ of PI589478 ('Novosibirski Sweet', M. domestica). Despite being highly variable, a trend line of bivariate function could be fit for fruit ethylene production $\left(r^{2}=0.120, P<0.0001\right.$, Figure 1a) and fruit firmness $\left(r^{2}=0.147, P<0.0001\right.$, Figure $\left.1 b\right)$.

The trend line of fruit ethylene showed a peak between days 10 and 15, which was largely a reflection of the mean fruit ethylene levels $75.5 \pm 100.5,207.3 \pm 193.9,272.8 \pm 249.6,247.0 \pm 170.8$ and $217.3 \pm 146.5\left(\mathrm{~nL} \mathrm{~g}^{-1} \mathrm{~h}^{-1}\right)$ at days $0,5,10,15$ and 20 , respectively (Figure 1a). A majority (59/97, 60.8\%) of the 97 Malus accessions reached their peak ethylene day at day 10 (25 accessions) or day 15 (34 accessions) while 2, 16 and 20 accessions topped their ethylene production at days 0,5 and 20 (Supplementary Figure S1c). The peak ethylene reads were spread from $1.7 \mathrm{~nL} \mathrm{~g}^{-1}$ per $\mathrm{h}$ of PI589570 (E36-7, M. hybrid) at day 20 to $1022.2 \mathrm{~nL} \mathrm{~g}^{-1}$ per $\mathrm{h}$ of PI633801 (M. sieversii) at day 10 (Supplementary Table S3).

As expected, fruit firmness showed a continuous decreasing trend during the 20-day period (Figure $1 \mathrm{~b}$ ). This was also an approximation of the mean firmness $7.4 \pm 1.7 \mathrm{~kg} \mathrm{~cm}^{-2}, 6.5 \pm$ $2.1 \mathrm{~kg} \mathrm{~cm}^{-2}, \quad 5.8 \pm 2.0 \mathrm{~kg} \mathrm{~cm}^{-2}, \quad 5.3 \pm 1.99 \mathrm{~kg} \mathrm{~cm}^{-2}$ and $5.3 \pm$ $1.92 \mathrm{~kg} \mathrm{~cm}^{-2}$ at days $0,5,10,15$ and 20 , respectively. In other words, the mean fruit firmness was lost by $13.6 \%$ at day $5,22.0 \%$ at day $10,29.2 \%$ at day 15 and $29.0 \%$ at day 20 .

Fruit ethylene production and firmness loss were significantly correlated (Table 1$)$. The strongest correlation $(r=0.564, P=0)$ was
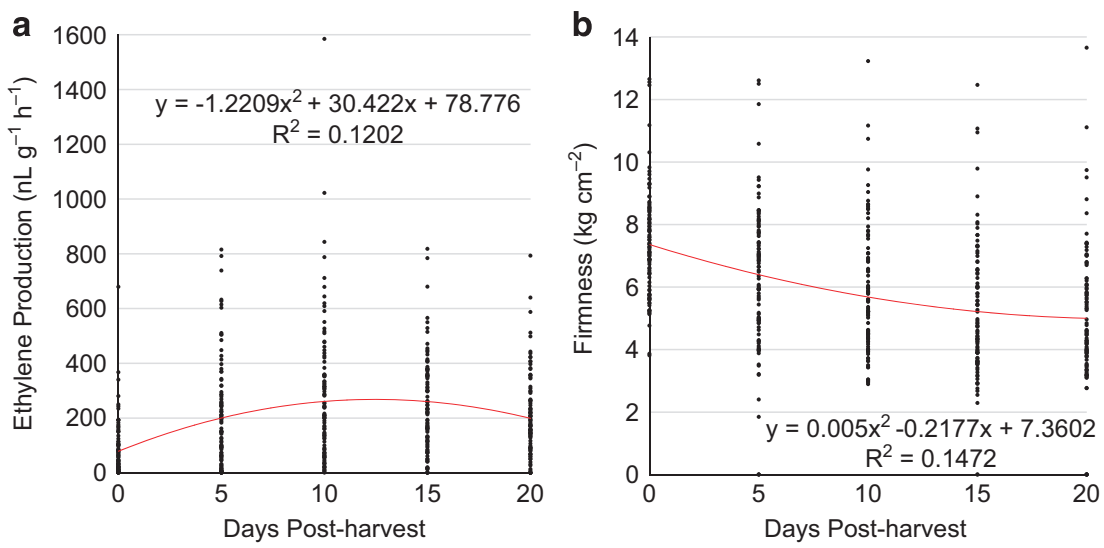

Figure 1. Evaluation of fruit ethylene production (a) and firmness (b) in 97 Malus accessions during a 20-day post-harvest period under room temperature. The trend lines (curves in red) and the associated equations and coefficient of determination $\left(R^{2}\right)$ are presented.

Table 1. Correlation coefficients between fruit ethylene production and firmness or firmness loss in 97 Malus accessions ${ }^{a}$

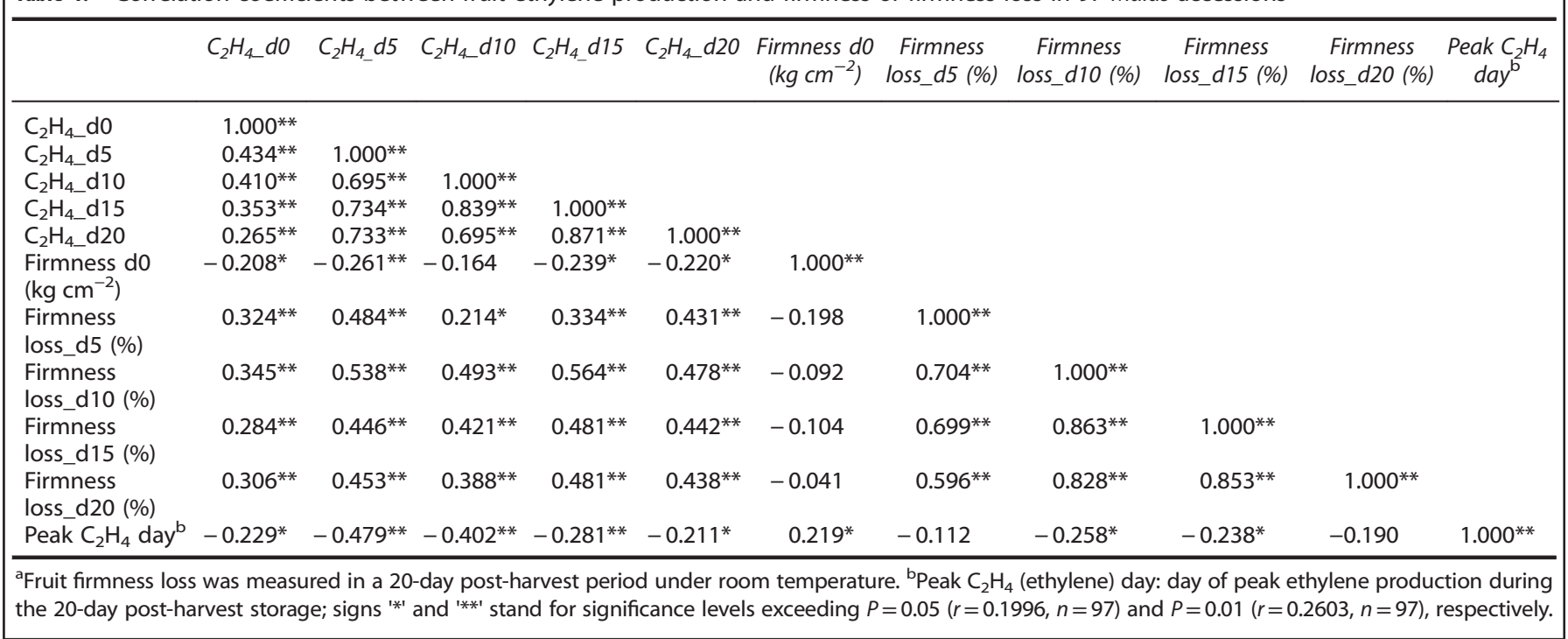




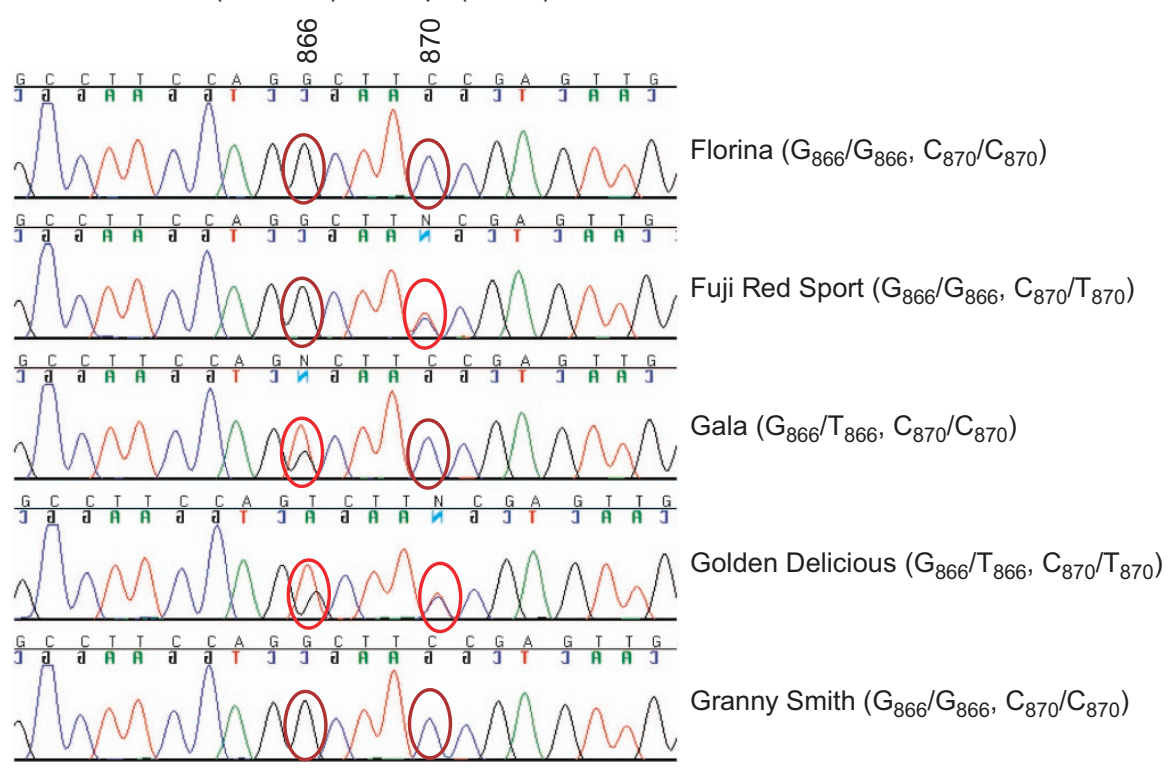

Figure 2. A chromatogram screenshot of the DNA sequence (partial) of MdACS3a encompassing SNPs $\mathrm{G}_{866} / \mathrm{T}_{866}$ and $\mathrm{C}_{870} / \mathrm{T}_{870}$ in six apple cultivars-'Florina', 'Fuji red sport', 'Gala', 'Golden Delicious' and 'Granny Smith'. The oval circles in brown and red indicate the homozygous or heterozygous status at the 866th and 870th nucleotides in the coding sequence of MdACS3a, respectively. The recognition sites of restriction enzymes Bst $\mathrm{NI}$ and $T a q^{\alpha} \mathrm{I}$ are provided to show that the mutation from $\mathrm{G}_{866}$ to $\mathrm{T}_{866}$ abolishes the restriction site of BstNI while the mutation from $\mathrm{C}_{870}$ to $\mathrm{T}_{870}$ gives rise to a restriction site for $T a q^{\alpha}$ l. The right panel shows allelotypes of $M d A C S 3 a$ as represented by the SNP alleles, where $\mathrm{G}_{866}$ stands for allele MdACS3a (wild type), $\mathrm{T}_{866}$ for MdACS3a-G289V (functional null allele), $\mathrm{C}_{870}$ also for allele MdACS3a and $\mathrm{T}_{870}$ for Mdacs3a (transcriptional null allele).

observed between ethylene at day 15 and fruit firmness loss at day 10 , while the weakest $(r=0.214, P=0.035)$ was between ethylene at day 10 and fruit firmness loss at day 5. Peak ethylene day (day of peak ethylene production during the 20-day postharvest storage) was most significantly correlated with ethylene at day $5(r=-0.479, P=6.9 \mathrm{E}-7)$, and it also significantly correlated with fruit firmness loss at day $10(r=-0.258, P=0.011)$ and day 15 $(r=-0.238, P=0.019)$ (Table 1$)$.

Development of allelic specific markers for MdACS3a

The null allele MdACS3a-G289V is caused by a mutation from $\mathrm{G}_{866}$ to $\mathrm{T}_{866}$ at the 866th base in the coding sequence of MdACS $3 a{ }^{18}$ Based on the web-based tool for single nucleotide polymorphism (SNP) analysis, ${ }^{25}$ the mutation abolishes the recognition site $\mathrm{CC}_{866} \mathrm{WGG}$ of restriction enzyme BstNI (Figure 2). To develop a CAPS marker, two primers (ACS3a-289F/R, Supplementary Table S2) were designed to amplify a DNA fragment $(480 \mathrm{bp})$ covering the $\operatorname{SNP}\left(\mathrm{G}_{866} / \mathrm{T}_{866}\right)$ specifically from MdACS3a although the three MdACS3 member genes MdACS3a (AB243060), MdACS3b (AB243061) and MdACS3C (AB243062) are of high identity in their DNA sequences. ${ }^{18}$ The specificity of the primer pair to MdACS3a was confirmed by sequencing of the PCR products from 92 of the 97 Malus accessions (Figure 2, Supplementary Table S3). Digestion of the PCR products with BstNI yielded restriction bands as expected (Figure $3 \mathrm{~b}$ ), indicating the successful development of a CAPS marker detecting SNP $\mathrm{G}_{866} / \mathrm{T}_{866}$, designated $\mathrm{CAPS}_{866}$. Therefore, allele $C A P S_{866} \mathrm{G}$ represents the wild-type allele MdACS3a while $C A P S_{866} T$ stands for the functional null allele MdACS3a-G289V.

Development of a marker detecting the transcriptional null allele Mdacs3a was initially thought to be challenging as the null allele was reported not to show sequence variations from the wild-type allele. ${ }^{18}$ However, sequencing analysis of the PCR products amplified by primers ACS3a-289F/R in the 92 accessions (Supplementary Table S3) not only identified the expected SNP
$\mathrm{G}_{866} / \mathrm{T}_{866}$, but also a new SNP $\mathrm{C}_{870} / \mathrm{T}_{870}$ (Figure 2 ). Importantly, this new SNP can discriminate the two alleles of MdACS3a in 'Fuji' (Figure 2), which was known of allelotype MdACS3a/Mdacs3a. ${ }^{18}$ Evidence from this and other studies (see Discussion) indicated that base $\mathrm{T}_{870}$ was associated with the Mdacs3a allele. Using a similar approach, another CAPS marker, named CAPS $_{870}$, was developed to detect SNP $\mathrm{C}_{870} / \mathrm{T}_{870}$ using restriction enzyme $T a q^{\mathrm{a}}$ । along with the same primers ACS3a-289F/R (Figure 3c). Therefore, allele $C A P S_{870} C$ corresponds to the wild-type allele MdACS3a while $\mathrm{CAPS}_{870} T$ corresponds to the transcriptional null allele Mdacs $3 a$.

Effect of the allelotypes of MdACS1 and MdACS3a on ethylene production and firmness loss

To evaluate the effect of the allelotypes of MdACS1 and MdACS3a, the 97 Malus accessions were assayed with markers ACS1, CAPS 866 and CAPS $_{870}$ that can detect different alleles of MdACS1 and MdACS3a (Figures 3a-c). As a result, marker ACS1 identified 53, 36 and 8 accessions of allelotypes of MdACS1-1/MdACS1-1 (MdACS11/1), MdACS1-1/MdACS1-2 (MdACS1-1/2) and MdACS1-2/MdACS12 (MdACS1-2/2), respectively (Supplementary Table S3). Similarly, marker CAPS $_{866}$ detected 75 accessions of allelotype $C A P S_{866} \mathrm{G}$ / $C^{C A P S_{866} G}\left(C A P S_{866} G / G\right), 18$ of $C A P S_{866} G / C A P S_{866} T\left(C A P S_{866} G / T\right)$ and 4 of $C A P S_{866} T / C A P S_{866} T\left(C A P S_{866} T / T\right)$; and marker $C A P S_{870}$ uncovered 47 accessions of allelotype $C A P S_{870} C / C^{2} A P S_{870} C\left(C A P S_{870} C / C\right)$,

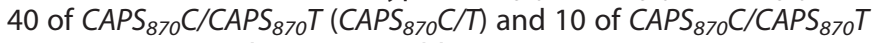
$\left(\right.$ CAPS $\left._{870} T / T\right)$ (Supplementary Table S3).

A series of one-way ANOVA of the fruit ethylene production and fruit firmness loss over the 20-day period within each of the three allelotype groups (Figure 4) indicated that the most differences were observed among the MdACS1 allelotypes. Allelotype MdACS1-1/1 showed significantly higher ethylene production (days 0-20) and firmness loss (days 5-20) than MdACS1-1/2 and MdACS1-2/2 allelotypes, but MdACS1-1/2 and MdACS1-2/2 did not differ in terms of ethylene production or firmness retention (Figures $4 a$ and d). In contrast, there were no difference among 
a

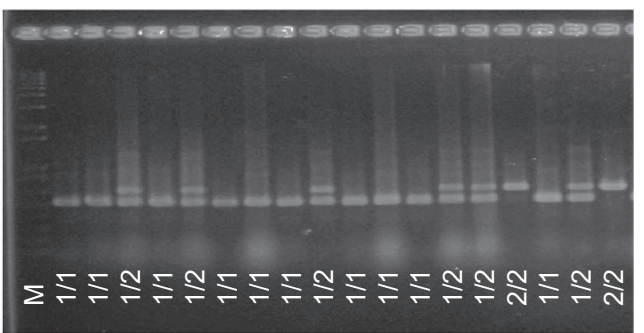

b
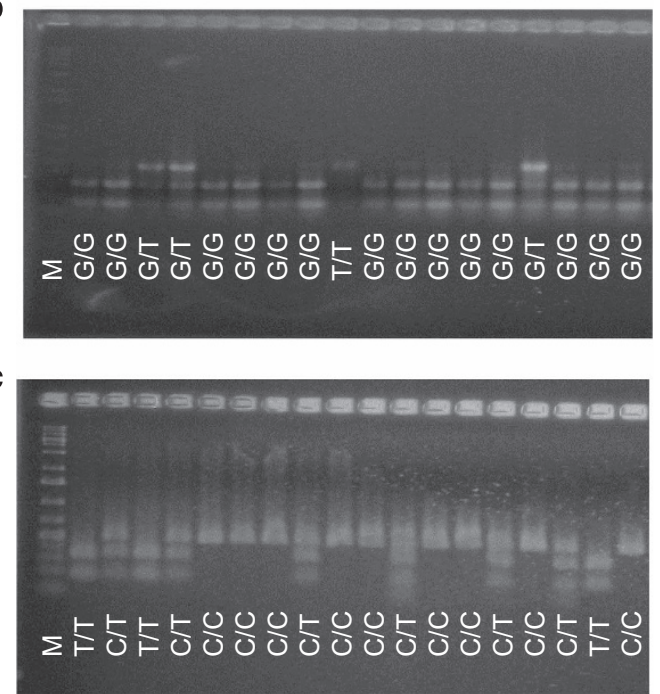

Figure 3. Agarose gel analyses of markers ACS1 (a), CAPS A66 (b) and $\mathrm{CAPS}_{870}(\mathbf{c})$. For marker ACS1, the PCR products amplified by primers ACS1-5F/R were directly analyzed. Allelotypes MdACS1-1/1, MdACS1$2 / 2$ and MdACS1-1/2 are denoted with ' $1 / 1$ ', ' $2 / 2$ ' and ' $1 / 2$ ' respectively. For marker $\mathrm{CAPS}_{866}$, the $\mathrm{PCR}$ products were first amplified by primers ACS3a-289F/R and then digested with enzyme $B s t \mathrm{NI}$, which restricts the $\operatorname{MdACS} 3 a\left(\mathrm{G}_{866}\right)$ allele into the two lower bands. Allelotypes MdACS3a/MdACS3a $\left(\mathrm{G}_{866} / \mathrm{G}_{866}\right)$, MdACS3a/ MdACS3a-G289V $\left(\mathrm{G}_{866} / \mathrm{T}_{866}\right)$ and MdACS3a-G289V/G289V ( $\left.\mathrm{T}_{866} / \mathrm{T}_{866}\right)$ are noted with ' $G / G$ ', 'G/T' and 'T/T', respectively. For marker $\mathrm{CAPS}_{870}$ enzyme $T a q^{\alpha}$ I restricts the Mdacs3a ( $\left.\mathrm{T}_{870}\right)$ allele into the two lower bands. Allelotypes MdACS3a/MdACS3a $\left(\mathrm{C}_{870} / \mathrm{C}_{870}\right), \operatorname{MdACS} 3 a /$ mdacs3a $\left(\mathrm{C}_{870} / \mathrm{T}_{870}\right)$ and $m d a c s 3 a / m d a c s 3 a\left(\mathrm{~T}_{870} / \mathrm{T}_{870}\right)$ are noted with ' $\mathrm{C} / \mathrm{C}^{\prime}$, 'C/T' and ' $\mathrm{T} / \mathrm{T}$ ', respectively.

the $\mathrm{CAPS}_{866}$ allelotypes in fruit ethylene production and firmness loss (Figures $4 \mathrm{~b}$ and e). Among the $\mathrm{CAPS}_{870}$ allelotypes, significant difference was not detected for ethylene production, but there were differences in fruit firmness loss between allelotypes $\mathrm{CAPS}_{870} \mathrm{C} / \mathrm{C}$ and $C A P S_{870} \mathrm{C} / T$ at day 5 and between $C A P S_{870} \mathrm{C} / \mathrm{C}$ and $C A P S_{870} T / T$ at day 10 (Figures $4 \mathrm{C}$ and $\mathrm{f}$ ). This indicated that such differences in fruit firmness loss at day 5 and 10 in the CAPS $_{870}$ allelotypes might be caused by other factors rather than their ethylene production levels.

To seek such factors, peak ethylene day, which measures ethylene peak timing, was examined (Figure 5) as this trait was negatively correlated with fruit firmness loss at day $10(r=-0.258$, $P=0.011)$ although the correlation was insignificant at day 5 $(r=-0.112, P=0.275)$ (Table 1). Encouragingly, the three CAPS $_{870}$ allelotypes showed significant difference from each other, with $C A P S_{870} \mathrm{C} / T$ having peaked the earliest, $C A P S_{870} \mathrm{C} / \mathrm{C}$ intermediate and $C A P S_{870} T / T$ the latest (Figure $5 a$ ). These data appeared to suggest that the earlier peak ethylene day of $C A P S_{870} \mathrm{C} / \mathrm{C}$ might have contributed to its greater fruit firmness loss of $C A P S_{870} C / C$ as compared with that of $C A P S_{870} T / T$ at day 10 (Figure 4f). However, the lowest fruit firmness loss of $C A P S_{870} C / T$ at day 5 remained to be explained. Peak ethylene day was also analyzed in the other two groups of allelotypes. In the allelotypes of MdACS1, MdACS1-1/ 1 had an earlier peak ethylene than MdACS1-2/2, but showed no difference from MdACS1-1/2 (Figure 5a). In the three allelotypes of $\mathrm{CAPS}_{866}$, no significant difference was observed (Figure 5a).

It was clear that the effect of MdACS1 on ethylene production and fruit firmness loss was much stronger than that of MdACS3a (Figure 4). To see if the random presence of the MdACS1 alleles might have obscured the detection of the effect of MdACS3a allelotypes (Figures $4 b, c$, e and f), another series of ANOVA was conducted for the MdACS3a allelotypes of five or more accessions (Figure 6) under the same background of MdACS1 allelotypes MdACS1-1/1 and MdACS1-1/2, which occurred in 53 and 36 of the 97 accessions (Supplementary Table S3), respectively. The third allelotype MdACS1-2/2 was not included in the analysis (Figure 6) due to limited number of 8 accessions.

For CAPS $_{866}$, the ANOVA analyses were conducted for two allelotypes $C A P S_{866} G / G$ and $C A P S_{866} G / T$ under MdACS1-1/1, as well as under MdACS1-1/2 (Figures $5 \mathrm{~b}$ and $6 \mathrm{a}, \mathrm{c}$ ). This allowed us to identify that allelotype $C A P S_{866} G / T$ produced significantly higher levels of ethylene than $C A P S_{866} G / G$ at day 10 under MdACS1-1/1 (Figure 6a). For $\mathrm{CAPS}_{870}$, three allelotypes $\mathrm{CAPS}_{870} \mathrm{C} / \mathrm{C}, \mathrm{CAPS}{ }_{870} \mathrm{C} / \mathrm{T}$ and $C A P S_{870} T / T$ under $M d A C S 1-1 / 1$ and two allelotypes $\mathrm{CAPS}_{870} \mathrm{C} / \mathrm{C}$ and $C A P S_{870} \mathrm{C} / T$ under $M d A C S 1-1 / 2$ were analyzed (Figures $5 \mathrm{c}$ and $6 \mathrm{~b}, \mathrm{~d}$ ). The results showed that allelotype $C A P S_{870} T / T$ had significant later peak ethylene day than $C A P S_{870} C / C$ and $C A P S_{870} C / T$ under MdACS1-1/1, and $C A P S_{870} C / C$ had significant later peak ethylene than $C A P S_{870} C / T$ under MdACS1-1/2 (Figure 5c). There were no significant differences detected between the other allelotypes of $\mathrm{CAPS}_{866}$ and $\mathrm{CAPS}_{870}$ at a given time point (Figures $5 b$ and $6 a-d$ ). These observations suggested that the direct effect of MdACS3a on ethylene production and firmness loss was limited, but its effect on peak ethylene day was clearly detectable through allele Mdacs3a $\left(\right.$ CAPS $\left._{870} T / T\right)$.

The analyses also provided information regarding the effect of

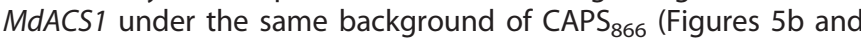
$6 a, c)$ or $\mathrm{CAPS}_{870}$ (Figures $5 \mathrm{c}$ and $6 \mathrm{~b}, \mathrm{~d}$ ) allelotypes. As expected, allelotype MdACS1-1/1 had higher ethylene production (Figures $6 a$ and c) and more firmness loss (Figures $6 \mathrm{~b}$ and d) than MdACS1$2 / 2$, but had similar peak ethylene day as MdACS1-1/2 (Figures $5 b$ and $c$ ) except under the $C A P S_{870} \mathrm{C} / \mathrm{C}$ background (Figure $5 \mathrm{c}$ ). These results suggested that the effect of MdACS1 on peak ethylene day was insignificant under the same background of MdACS3a, which was in disagreement with the observation that the effect of MdACS1 on peak ethylene day was significant when the background of MdACS3a was not considered (Figure $5 a$ ).

Since the MdACS3a allelotype CAPS $_{866} T / T$ (MdACS3a-G289V/ G289V was present only in 4 of 97 accessions, the 2 controlled crosses GMAL4592 and GMAL4593 segregating for CAPS 866 T/T under the same background of MdACS1-1/2 were used for better analysis. In total, 17 progeny of allelotype $C A P S_{866} G / G(M d A C S 3 a /$ MdACS3a) and another 17 of $C A P S_{866} T / T$ were similarly evaluated for ethylene production and fruit firmness loss. ANOVA analysis indicated that there were no significant differences between the two allelotypes $C A P S_{866} G / G$ and $C A P S_{866} T / T$ in ethylene production and fruit firmness loss, nor in peak ethylene day from day 0 to day 20 (Supplementary Figures $2 \mathrm{a}-\mathrm{c}$ ), suggesting that no effect of allelotype CAPS $_{866} T / T$ (MdACS3a-G289V/G289V) was detectable in this study.

\section{Allelotyping of MdACS1 and MdACS3a in a large set of Malus} accessions

Additional 855 Malus accessions were surveyed with markers ACS1, CAPS 866 and CAPS $_{870}$, leading to a total of 952 Malus accessions allelotyped (Figure 7, Supplementary Table S1). The data showed that the three allelotypes MdACS1-1/1, MdACS1-1/2 


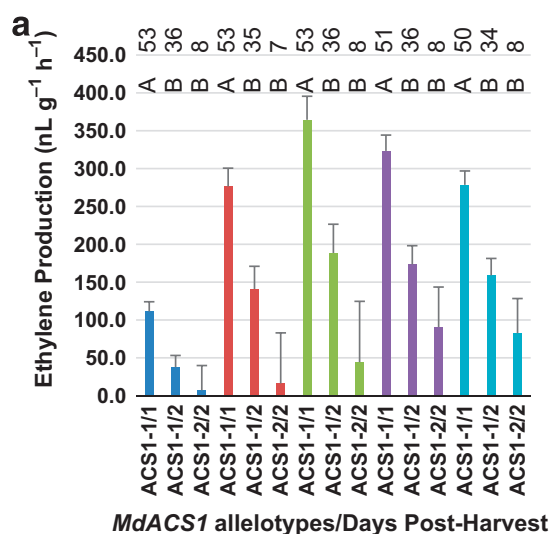

d

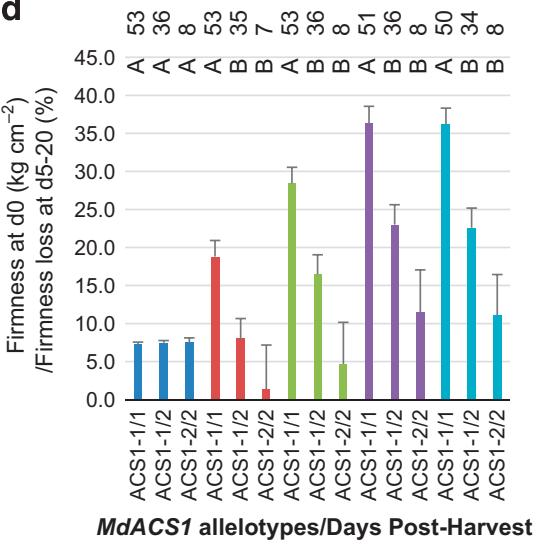

b

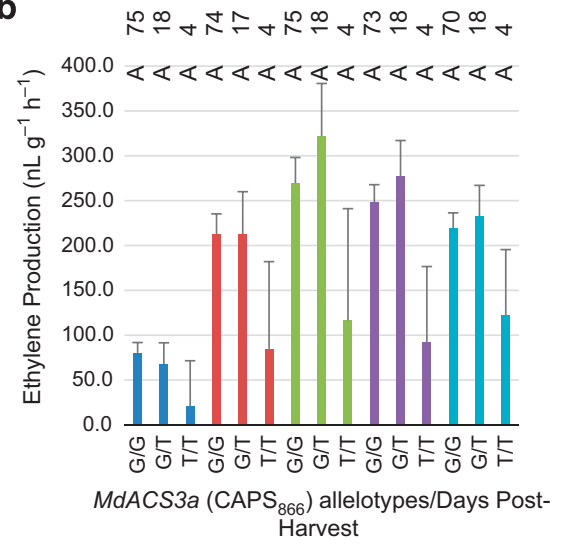

e

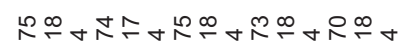

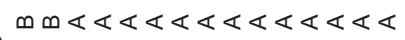

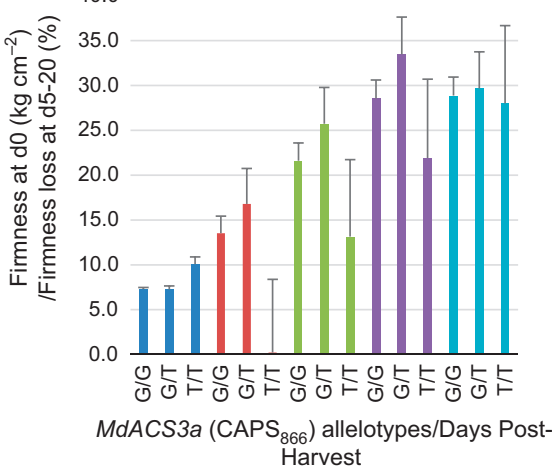

c

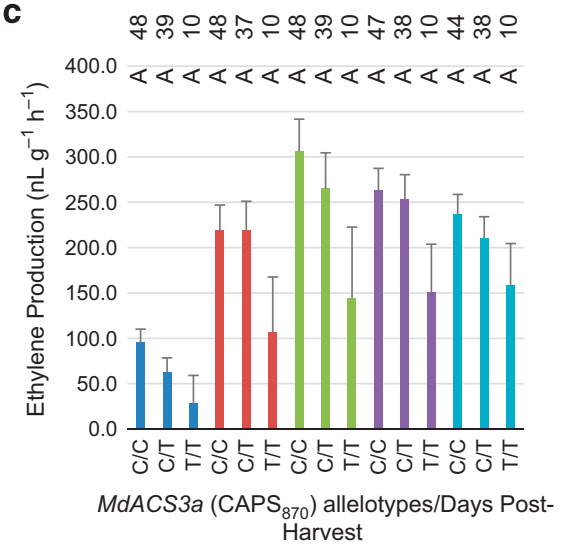

f

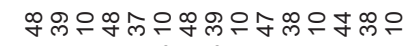

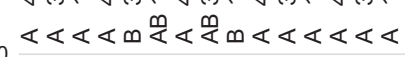

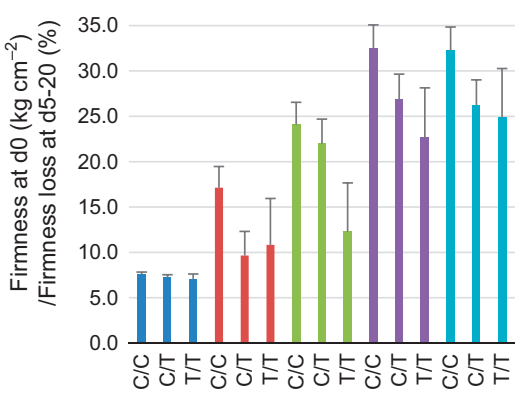

MdACS3a $\left(\mathrm{CAPS}_{870}\right)$ allelotypes/Days PostHarvest

Figure 4. Comparison of the means of fruit ethylene production and firmness or firmness loss among allelotypes of MdACS1 as defined by marker ACS1 (a and $\mathbf{d}$ ), and among those of MdACS3a as defined by markers CAPS 866 (b and $\mathbf{e})$ and CAPS 870 (c and $\mathbf{f})$. The allelotypes are annotated similarly as those in the legend of Figure 3. Colors of column in blue, orange, green, purple and turquoise represent days 0,5 , 10,15 and 20, respectively. The statistical tests were conducted independently within each of the five storage time points (days $0-20$ ). Significance levels are indicated with letters (shown above the columns in the chart), where different letters indicate $P<0.05$. The numbers of accessions observed $(n)$ for each allelotype are presented accordingly (shown above the letters for significance). Error bars indicate s.e.

a

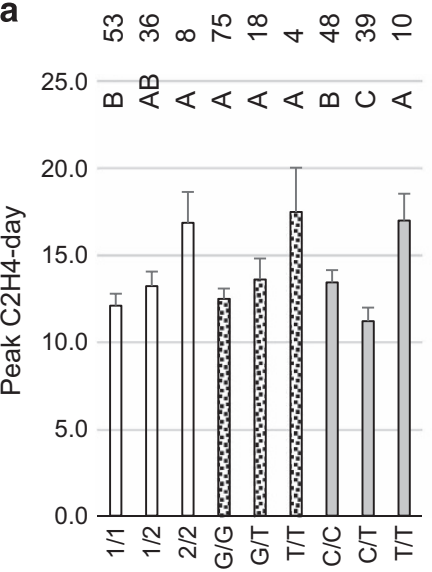

Allelotypes of MdACS1, MdACS3a

$\left(\mathrm{CAPS}_{866}\right)$, and MdACS3a $\left(\mathrm{CAPS}_{870}\right)$ b

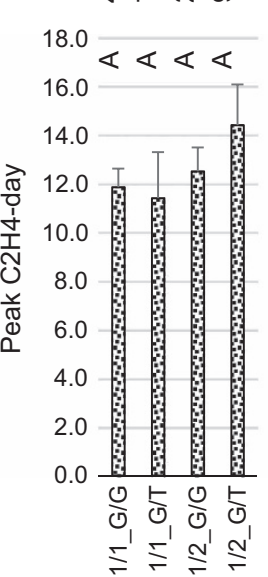

Allelotypes of MdACS3a

$\left(\mathrm{CAPS}_{866}\right)$ under

MdACS $1-1 / 1$ or $-1 / 2$
C

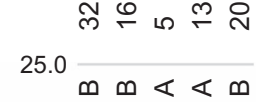

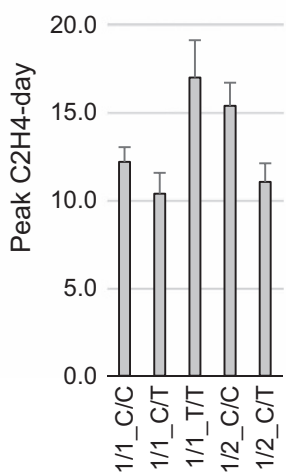

Allelotypes of MdACS3a

(CAPS $_{870}$ ) under MdACS1$1 / 1$ or $-1 / 2$

Figure 5. Comparison of the means of peak ethylene day among the allelotypes of MdACS1 as defined by marker ACS1 (open column) and those of MdACS3a as defined by markers CAPS 866 (dot-filled column) and CAPS ${ }_{870}$ (filled column) (a), and among the allelotypes of MdACS3a defined by markers CAPS $_{866}$ (b) and CAPS $_{870}$ (c) under the same background of MdACS1-1/1 or MdaCS1-1/2. The allelotypes, significance levels and observed numbers are represented similarly as those in Figures 3 and 4. 
and MdACS1-2/2 were of 665,249 and 38 accessions, the allelotypes $C A P S_{866} G / G, C A P S_{866} G / T$ and $C A P S_{866} T / T$ were of 770 , 173 and 9 accessions, and the allelotypes $C A P S_{870} C / C, C A P S_{870} C / T$ and $C A P S_{870} T / T$ were of 346,400 and 206 accessions, respectively. Estimating the allele frequency in the 952 accessions revealed alleles MdACS1-1 and MdACS1-2 of $82.9 \%$ and $17.1 \%$, CAPS $_{866} G$

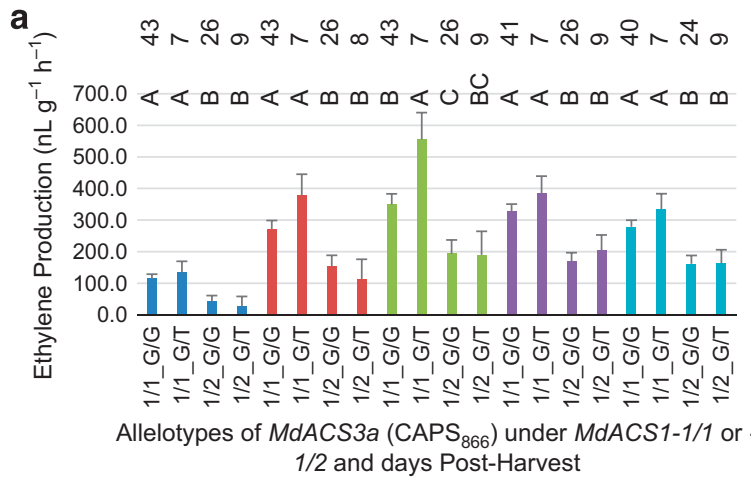

C

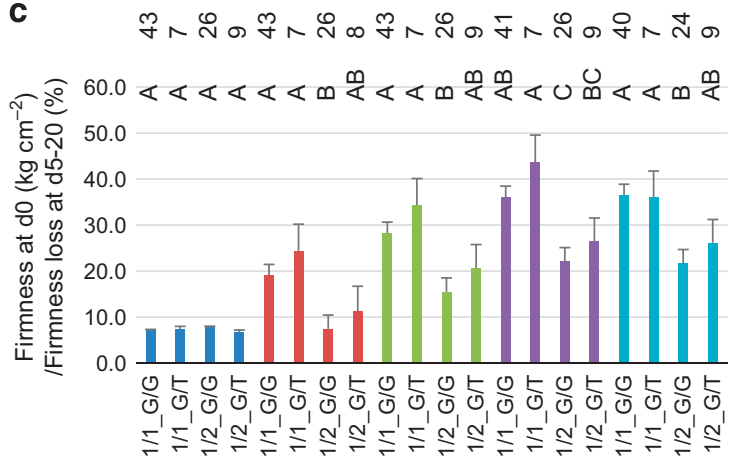

Allelotypes of MdACS3a (CAPS $_{866}$ ) under MdACS1-1/1 or $-1 / 2$ and days Post-Harvest

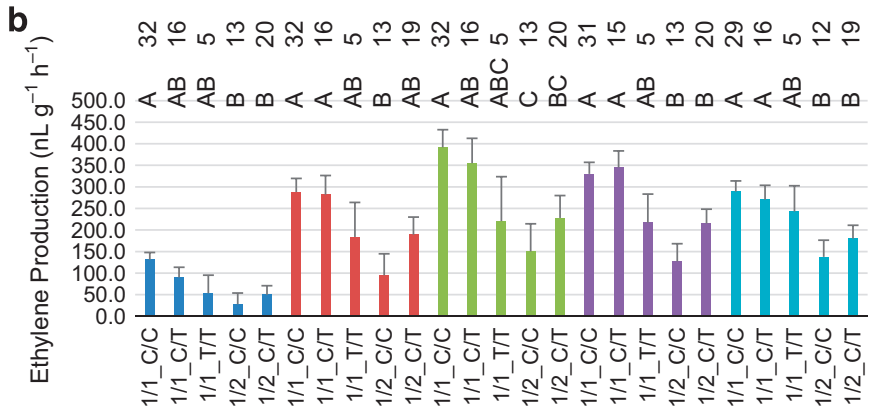

Allelotypes of MdACS3a (CAPS $_{870}$ ) under MdACS1-1/1 or $-1 / 2$ and days Post-Harvest

d

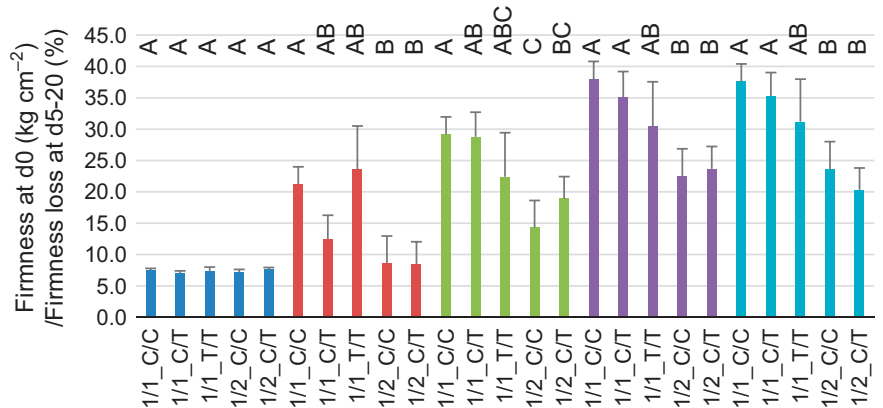

Allelotypes of MdACS3a (CAPS $_{866}$ ) under MdACS1-1/1 or $-1 / 2$ and days Post-Harvest

Figure 6. Comparison of the means of ethylene production and fruit firmness or firmness loss among the allelotypes of MdACS3a as defined by markers CAPS 866 (a and $\mathbf{c}$ ) and CAPS 870 (b and $\mathbf{d})$ under the same background of MdACS1-1/1 or MdaCS1-1/2. The allelotypes, column colors, statistical tests, significance levels and observed numbers are represented similarly as those in Figures 3 and 4.

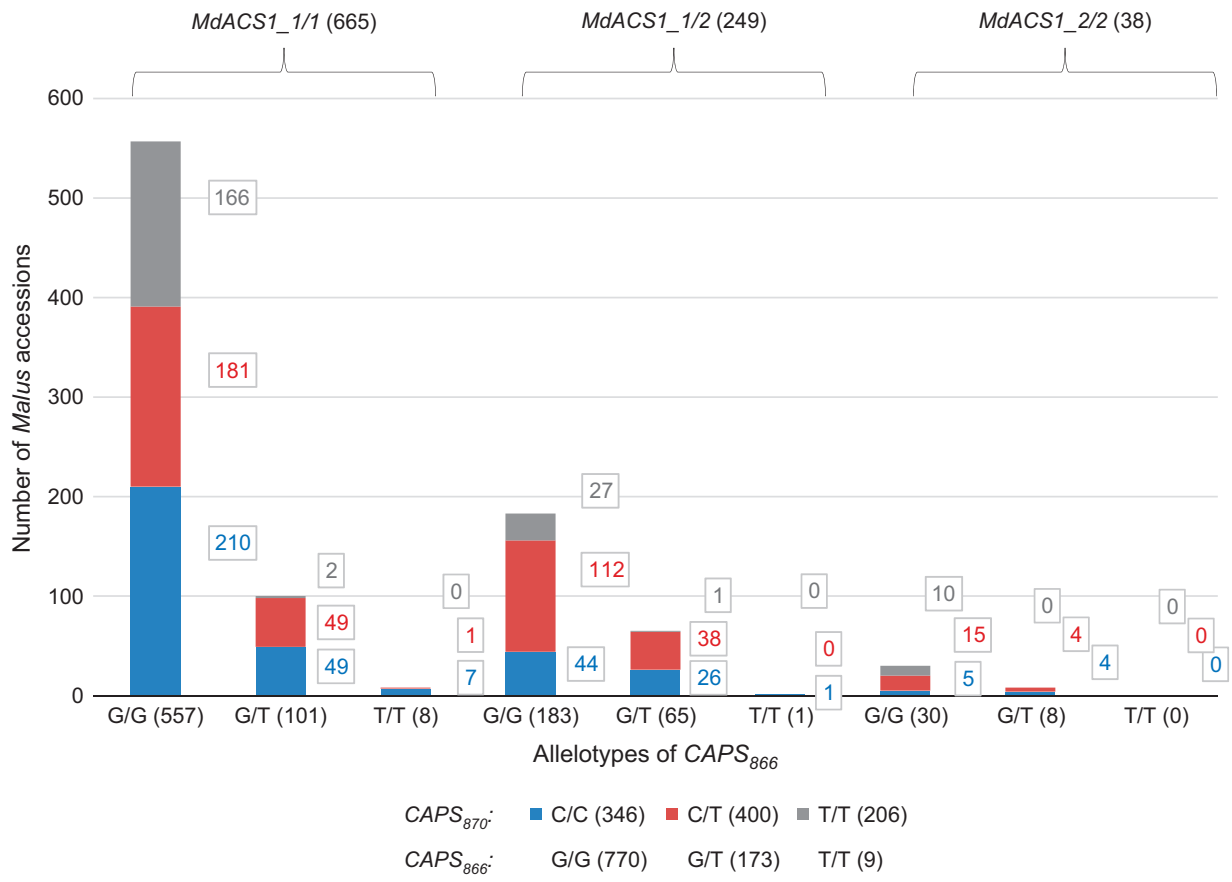

Figure 7. Allelotyping of MdACS1 and MdACS3a using markers ACS1, CAPS 866 and CAPS $_{870}$ in 952 Malus accessions. The numbers in parentheses stand for the total or subtotal number of Malus accessions in an allelotype proximately annotated. The allelotypes are represented similarly as those in Figure 3. 
a Overall, $n=952$

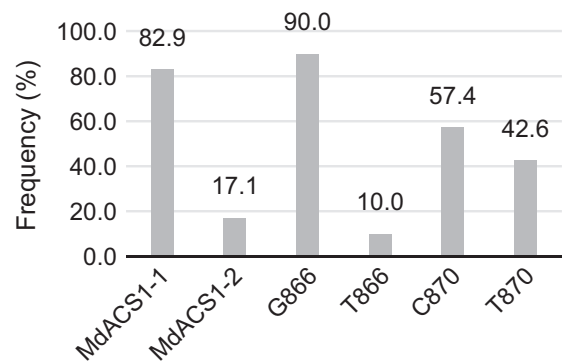

Alleles of MdACS1 and MdACS3a (CAPS $_{866}$ and CAPS 870 )

C M. Hybrid, $\mathrm{n}=146$

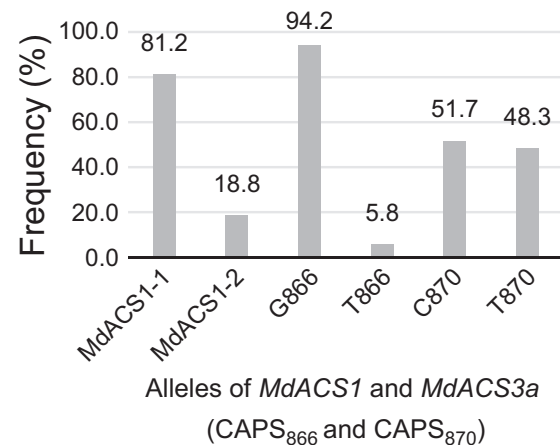

b M. domestica, $\mathrm{n}=508$

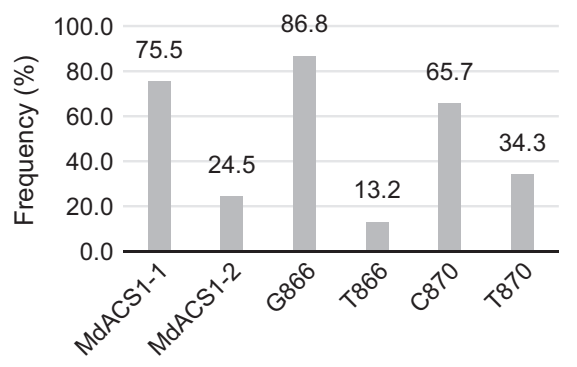

Alleles of MdACS1 and MdACS3a (CAPS $_{866}$ and CAPS $_{870}$ )

d $M$. sieversii, $\mathrm{n}=78$

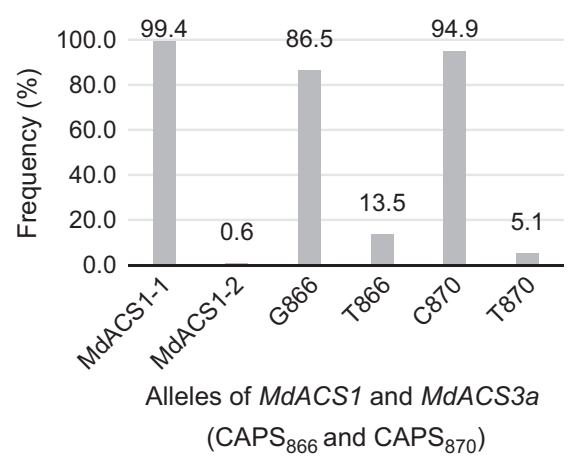

Figure 8. Frequency of the MdACS1 and MdACS3a alleles as defined by markers ACS1, CAPS 866 and CAPS 870 in all the 952 Malus accessions (a), M. domestica (b), M. hybrid (c) and M. sieversii (d).

and CAPS $_{866} T$ of $90.0 \%$ and $10.0 \%$, and CAPS $_{870} \mathrm{C}$ and $C A P S_{870} T$ of $57.4 \%$ and $42.6 \%$, respectively (Figure 8 a).

To investigate whether and how human selection might have favored or repressed these alleles, their frequency in the most represented species $M$. domestica (508 accessions), M. hybrid (146) and $M$. sieversii (78), which collectively accounted for $76.9 \%$ of the 952 accessions (Supplementary Table S1), were independently estimated (Figures $8 \mathrm{~b}-\mathrm{d}$ ). In comparison with $M$. sieversii, $M$. domestica and $M$. hybrid showed the largest allele frequency increases for alleles MdACS1-2 (from $0.6 \%$ to $18.8-24.5 \%$ ) and CAPS $_{870} T$ (from $5.1 \%$ to $34.3-48.3 \%$ ), or decreases for allele MdACS1-1 (from 99.4\% to 81.2-75.5\%) and CAPS $_{870} \mathrm{C}$ (from $94.9 \%$ to $65.7-51.7 \%$ ), but minimal changes for the $C A P S_{866} G$ (from $86.5 \%$ to $86.8-94.2 \%$ ) and $C^{-1 P S_{866}}$ T (from $13.5 \%$ to $13.2-5.8 \%$ ) alleles (Figures $8 \mathrm{~b}-\mathrm{d}$ ). These results suggested that apple-breeding practice may have selected for alleles MdACS1-2 and $C A P S_{870} T$ (Mdacs3a), against alleles MdACS1-1 and $C A P S_{870} C$, and is neutral for alleles $C A P S_{866} G$ and $C A P S_{866} T$ (MdACS3a-G289V). Such human selection for alleles MdACS1-2 and Mdacs3a supported their observed significant effect on reduced or delayed ethylene production. Meanwhile, the minimal changes in the frequency of allele MdACS3a-G289V reinforced the unfound effect of this allele on ethylene.

\section{DISCUSSION}

The effect of MdACS1 and MdACS3a and beneficial alleles

The allelic effect of MdACS1 on fruit ethylene production and softening was significant and detectable at nearly all time points tested during the 20-day post-harvest period in the 97 Malus accessions. This was consistent with the critical role of MdACS1 reported in many other studies. ${ }^{10-16,26-29}$ Since the allele frequency of MdACS1-2 was $24.5 \%$ in $M$. domestica, $18.8 \%$ in M. hybrid and only $0.6 \%$ in M. sieversii (Figure 8 ), which is the major progenitor species of domestic apples, artificial selection has clearly favored MdACS1-2 over MdACS1-1. In fact, such allele preference of MdACS1-2 over MdACS1-1 was even reported within $M$. domestica when the frequencies of the two alleles in apple cultivars were plotted against their time of introduction. ${ }^{16}$ These observations are in accordance with the finding that allele MdACS1-2 is a beneficial allele associated with low ethylene and slow softening (Figures 4 and 6).

MdACS3a was regarded a main regulator for ethylene production transition from system 1 to $2 .^{18}$ The gene was also similarly shown to be an accelerator ${ }^{30}$ or an inducer $^{31}$ of apple fruit ripening based on its gene expression timing and patterns in apple cultivars of varying ethylene levels and softening rates. In this study, such roles of MdACS3a were also detected through examining the allelic effect of Mdacs3a $\left(C A P S_{870} T\right)$ on peak ethylene day, which reflects the timing of the climacteric ethylene burst. For example, under the same background of MdACS1-1/1, allelotype Mdacs3a/Mdacs3a $\left(\mathrm{CAPS}_{870} \mathrm{~T} / \mathrm{T}\right)$ showed a significant delay in peak ethylene day when compared with what was observed for allelotypes MdACS3a/MdACS3a (CAPS $\left.{ }_{870} G / G\right)$ and MdACS3a/Mdacs3a (CAPS $870 G / T$ ) (Figure 5c). Moreover, the allele frequency of Mdacs $3 a$ (CAPS $\left._{870} T\right)$ was $34.3 \%$ in $M$. domestica and $48.3 \%$ in $M$. hybrid, a dramatic increase from the corresponding frequency of $5.1 \%$ in $M$. sieversii, indicating a strong human selection for allele Mdacs3a, presumably for the benefit of delayed ethylene production. Taken together, these data support the regulatory role of $M d A C S 3 a$ in ethylene production transition in apple fruit.

However, the allelic effect of MdACS3a-G289V on fruit ethylene production, softening and peak ethylene day was shown to be insignificant in the 97 Malus accessions, as well as in the 34 
progeny from the 2 controlled crosses segregating for allelotype MdACS3a-G289V/G289V (CAPS $\left.{ }_{866} T / T\right)$ under the same background of MdACS1 allelotype. Furthermore, the allele frequency of MdACS3a-G289V (CAPS $_{866}$ T) was $13.5 \%$ in M. sieversii, $13.2 \%$ in $M$. domestica and $5.8 \%$ in $M$. hybrid, providing no evidence that MdACS3a-G289V $\left(\right.$ CAPS $\left._{866} T\right)$ has been enriched in response to selection. These results were surprising as MdACS3a-G289V was shown to be a functional null allele of MdACS3a. ${ }^{18}$ In a previous study, the two null alleles MdACS3a-G289V (CAPS $\left.{ }_{866} T\right)$ and Mdacs3a $\left(C A P S_{870} T\right)$ were concluded to affect the ripening initiation only in late-season apple cultivars, but not in early- or mid-season ones. ${ }^{20}$ Such discrepancy in different studies regarding the roles of the two null alleles of MdACS3a, particularly MdACS3a-G289V, calls for further investigations into the role of MdACS3a-G289V. Nevertheless, alleles MdACS1-2 and Mdacs3a $\left(\right.$ CAPS $\left._{870} T\right)$ are clearly demonstrated to be beneficial for breeding apples of low or delayed ethylene profiles in this study, a first effort that simultaneously assessed the roles of MdACS1 and MdACS3a in fruit ethylene production and softening in highly diverse Malus materials.

\section{Markers ACS1, $\mathrm{CAPS}_{866}$ and $\mathrm{CAPS}_{870}$}

The assessment of the roles of MdACS1 and MdACS3a in apple fruit ethylene production and softening largely relied on the previously developed marker ACS1 ${ }^{10,11}$ and the two markers $\mathrm{CAPS}_{866}$ and CAPS $_{870}$ developed in this study. Since CAPS $_{866}$ directly detects the mutation SNP $\mathrm{G}_{866} / \mathrm{T}_{866}, \mathrm{CAPS}_{866}$ is an unequivocal marker for identifying the functionally null allele MdACS3a-G289V. ${ }^{18}$ Marker CAPS $_{870}$ detects SNP $C_{870} / T_{870}$ that does not correspond to a change in the encoding amino acid, that is, CAPS $_{870}$ detects a silent mutation in MdACS3a. Regardless of the nature of SNP $\mathrm{C}_{870}$ / $\mathrm{T}_{870}, \mathrm{~T}_{870}$ is a genetic signature for allele Mdacs $3 a$ as the mutation was identified in 'Fuji', the very source from which the transcriptional null allele Mdacs $3 a$ was originally defined. ${ }^{18}$ Based on the genomic DNA sequences from 'Fuji', alleles MdACS3a (JF833309) and Mdacs3a (JF833309) differ by 14 nucleotides, and of these, only 4 were within the coding sequence. ${ }^{20}$ Sequencing of the 92 Malus accessions in this study indicated that SNP $\mathrm{C}_{870} / \mathrm{T}_{870}$ is authentic and varying only between 2 nucleotides $C_{870}$ and $T_{870}$ (Figure 2, Supplementary Table S3). These data strongly support that $\mathrm{CAPS}_{870}$ is a reliable marker for detecting allele Mdacs3a. Since both CAPS $_{866}$ and CAPS $_{870}$ detect the characterized SNPs in the coding sequence of MdACS3a and can be simply performed by electrophoresis on agarose gels, the two markers are readily applicable for marker-assisted selection in apple breeding.

Since SNP $\mathrm{C}_{870} / \mathrm{T}_{870}$ is located only four bases downstream of SNP $\mathrm{G}_{866} / \mathrm{T}_{866}$, markers CAPS $_{866}$ and CAPS $_{870}$ were once considered to be used as a single marker in this study. However, such usage would lead to an ambiguous scenario for allelotype $\mathrm{G}_{866} \mathrm{~T}_{866} / \mathrm{C}_{870} \mathrm{~T}_{870}$ as it could be formed by a combination either between gametes $G_{866} T_{870}$ and $T_{866} C_{870}$ or between gametes $\mathrm{G}_{866} \mathrm{C}_{870}$ and $\mathrm{T}_{866} \mathrm{~T}_{870}$. To avoid such possible uncertainty, the two markers were used independently.

Previously, an SSR marker targeting at the promoter region of MdACS3a was developed and used to allelotype MdACS3a in 103 apple varieties. ${ }^{20}$ It was shown that three alleles (331, 353, and $359 \mathrm{bp}$ ) of the SSR marker corresponded to the wild-type allele MdACS3a (that is, MdACS3a-1 in ref. 20), two alleles (333 and $335 \mathrm{bp}$ ) to Mdacs3a (that is, MdACS3a-2) and one allele (361 bp) to MdACS3a-G289V (that is, MdACS3a-1V). This makes the corresponding relationship between the SSR marker alleles and the MdACS3a alleles somewhat indirect and inconvenient. Since the size of the SSR marker alleles frequently differ by $2 \mathrm{bp}$, an automatic DNA sequencer-based detection system is necessary, thereby requiring more sophisticated handling and analysis, compared with the agarose gel-based markers CAPS $_{866}$ and CAPS 870 . However, identical allelotypes were observed for all 19 apple cultivars used by co-insistence in both studies (Supplementary Table S4), suggesting that the SSR marker and the 2 CAPS markers are useful for allelotyping of MdACS3a. As expected, identical allelotypes for MdACS1 were also obtained for the 19 common apple cultivars between these 2 studies (Supplementary Table S4).

It should be mentioned that two degenerated CAPS (dCAPS) markers were developed to confirm alleles Mdacs $3 a$ and MdACS3a-G289V in CDNA, but the two dCAPS markers were not used for allelotyping the MdACS3a alleles. ${ }^{20}$ Therefore, the applicability of the dCAPS markers is unknown in diverse apples.

Utility of the data

Of the 952 Malus accessions, 97 were evaluated for their fruit ethylene production and softening at 5 time points over a 20-day post-harvest period (Supplementary Table S3). Although most accessions seemed to have predictable ethylene-regulated postharvest behaviors, 'Virginia Gold' (PI588778, M. domestica) was unusual as it had minimal firmness loss (comparable to 'Fuji') during the 20-day storage while producing high levels of ethylene (comparable to 'Golden Delicious'). This suggested that the slow softening (long-shelf life) character of 'Virginia Gold' is likely less dependent on ethylene production. More importantly, 'Virginia Gold' has also been shown with an excellent storability. ${ }^{32}$ To understand the lack of ethylene-related softening in 'Virginia Gold', several preliminary experiments have been initiated by the authors. In melon, it was reported that flesh softening involved both ethylene-dependent and -independent components. ${ }^{33}$ In tomato, the ethylene-independent aspects of fruit ripening were evidenced to be regulated by the FRUITFULL homologs. ${ }^{34}$ It is possible that investigating fruit softening independent of or less dependent on ethylene production would lead to new knowledge for better understanding of the apple fruit-ripening process, promising an interesting research area in apple post-harvest biology.

In addition, the data set of allelotypes for genes MdACS1 and MdACS3a generated in the 952 Malus accessions would be useful for other future studies involving MdACS1 and MdACS3a, which are the only 2 apple ACS genes known to be expressed specifically in fruit and associated with apple fruit ethylene production and firmness. ${ }^{8,9,13}$ The data set, together with three markers ACS1, $\mathrm{CAPS}_{866}$ and $\mathrm{CAPS}_{870}$, would be also useful for planning new crosses for developing improved apples with low ethylene and reduced loss of firmness.

\section{Usage of terms allelotype and allelotyping}

Term allelotype is defined as 'the frequency of alleles in a breeding population.' according to 'A Dictionary of Genetics'. ${ }^{35}$ In this study, allelotype is referred to the allele composition at a specific gene locus, that is, MdACS1 or MdACS3a, in individual accessions, highly similar to term 'genotype' for a given DNA marker. Such usage of allelotype represents a drift from or an expansion for the original definition of allelotype defined in the dictionary. However, the usage offers convenience for describing allele composition at a specific gene locus. Indeed, such usage has been adapted already in literature. ${ }^{14,18,20}$

The definition for term allelotyping in "Encyclopedia of Genetics, Genomics, Proteomics, and Informatics' ${ }^{136}$ reads 'Allelotyping is the determination of the spectrum and frequency of allelic variations in a population.' The usage of allelotyping in this study is largely covered by the definition, but an extension to include activities for determining allelotype (allele composition at a specific gene locus) is also practiced.

\section{Conclusions}

A substantial effort to simultaneously assess the roles of MdACS1 and MdACS3a in fruit ethylene production and softening in diverse 
Malus materials is presented in this study. The most relevant findings include: (1) MdACS1 had much greater direct influence on fruit ethylene production and softening than MdACS3a. (2) Allele MdACS1-2 was associated with low ethylene and slow softening while MdACS1-1 with high ethylene and rapid softening. (3) Under the same background of MdACS1 allelotypes, the transcriptional null allele Mdacs3a, rather than the functional null allele ACS3a$G 289 \mathrm{~V}$, significantly delayed the time required to reach the climacteric ethylene peak. (4) Alleles MdACS1-2 and Mdacs3a, but not ACS3a-G289V, were highly enriched in $M$. domestica and M. hybrid when compared with those in the M. sieversii. Overall, this study provides important information as to which alleles of MdACS1 and MdACS3a are beneficial for low and delayed ethylene production and how these beneficial alleles can be selected for apple improvement.

\section{CONFLICT OF INTEREST}

The authors declare no conflict of interest.

\section{ACKNOWLEDGEMENTS}

This work was financially supported in part by the National Plant Germplasm System (NPGS), Apple Crop Germplasm Committee (CGC), Federal Formula Funds, and College of Agriculture and Life Science, Cornell University.

\section{REFERENCES}

1 Burmeister DM, Dilley DR. A 'scald-like' controlled atmosphere storage disorder of Empire apples-a chilling injury induced by $\mathrm{CO}_{2}$. Postharvest Biol Technol 1995; 6: $1-7$.

2 Watkins CB, Silsby KJ, Goffinet MC. Controlled atmosphere and antioxidant effects on external $\mathrm{CO}_{2}$ injury of 'Empire' apples. HortScience 1997; 32: 1242-1246.

3 Watkins CB, Erkan M, Nock JF, lungerman KA, Beaudry RM, Moran RE. Harvest date effects on maturity, quality, and storage disorders of 'Honeycrisp' apples. HortScience 2005; 40: 164-169.

4 Fawbush F, Nock JF, Watkins CB. External carbon dioxide injury and 1-methylcyclopropene (1-MCP) in the 'Empire' apple. Postharvest Biol Technol 2008; 48: 92-98.

5 Yang SF, Hoffman NE. Ethylene biosynthesis and its regulation in higher-plants. Annu Rev Plant Phys 1984; 35: 155-189.

6 Barry CS, Giovannoni JJ. Ethylene and fruit ripening. J Plant Growth Regul 2007; 26: 143-159.

7 Cara B, Giovannoni JJ. Molecular biology of ethylene during tomato fruit development and maturation. Plant Sci 2008; 175: 106-113.

8 Kondo S, Meemak S, Ban Y, Moriguchi T, Harada T. Effects of auxin and jasmonates on 1-aminocyclopropane-1-carboxylate (ACC) synthase and ACC oxidase gene expression during ripening of apple fruit. Postharvest Biol Technol 2009; 51: 281-284.

9 Wiersma PA, Zhang H, Lu C, Quail A, Toivonen PMA. Survey of the expression of genes for ethylene synthesis and perception during maturation and ripening of 'Sunrise' and 'Golden Delicious' apple fruit. Postharvest Biol Technol 2007; 44: 204-211.

10 Harada T, Sunako T, Wakasa Y, Soejima J, Satoh T, Niizeki M. An allele of the 1aminocyclopropane-1-carboxylate synthase gene (Md-ACS1) accounts for the low level of ethylene production in climacteric fruits of some apple cultivars. Theor Appl Genet 2000; 101: 742-746.

11 Sunako R, Sakuraba W, Senda M, Akada S, Ishikawa R, Niizeki M et al. An allele of the ripening-specific 1-aminocyclopropane-1-carboxylic acid synthase gene (ACS1) in apple fruit with a long storage life. Plant Physiol 1999; 119: 1297-1303.

12 Oraguzie NC, Iwanami H, Soejima J, Harada T, Hall A. Inheritance of the Md-ACS1 gene and its relationship to fruit softening in apple (Malus $\mathrm{x}$ domestica Borkh.). Theor Appl Genet 2004; 108: 1526-1533.

13 Costa F, Peace CP, Stella S, Serra S, Musacchi S, Bazzani M et al. QTL dynamics for fruit firmness and softening around an ethylene-dependent polygalacturonase gene in apple (Malus x domestica Borkh.). J Exp Bot 2010; 61: 3029-3039.

14 Sato T, Kudo T, Akada T, Wakasa Y, Niizeki M, Harada T. Allelotype of a ripeningspecific 1-aminocyclopropane-1-carboxylate synthase gene defines the rate of fruit drop in apple. J Am Soc Hortic Sci 2004; 129: 32-36.
15 Zhu Y, Barritt B. Md-ACS1 and Md-ACO1 genotyping of apple (Malu x domestica Borkh.) breeding parents and suitability for marker-assisted selection. Tree Genet Genomes 2008; 4: 555-562.

16 Nybom H, Sehic J, Garkava-Gustavsson L. Modern apple breeding is associated with a significant change in the allelic ratio of the ethylene production gene Md-ACS1. J Hort Sci Biotechnol 2008; 83: 673-677.

17 Rosenfield C-L, Kiss E, Hrazdina G. MdACS-2 (Accession No. U73815) and MdACS-3 (Accession No. U73816): Two new I-aminocyclopropane-1-carboxylate synthases in ripening apple fruit. Plant Physiol 1996; 112: 1735-1736.

18 Wang A, Yamakake J, Kudo H, Wakasa Y, Hatsuyama Y, Igarashi M et al. Null mutation of the MdACS3 gene, coding for a ripening-specific 1-aminocyclopropane1-carboxylate synthase, leads to long shelf life in apple fruit. Plant Physiol 2009; 151: 391-399.

19 Boualem A, Fergany M, Fernandez R, Troadec C, Martin A, Morin H et al. A conserved mutation in an ethylene biosynthesis enzyme leads to andromonoecy in melons. Science 2008; 321: 836-838.

20 Bai S, Wang A, Igarashi M, Kon T, Fukasawa-Akada T, Li T et al. Distribution of MdACS3 null alleles in apple (Malus $\mathrm{x}$ domestica Borkh.) and its relevance to the fruit ripening characters. Breed Sci 2012; 62: 46-52.

21 Forsline PL, Aldwinckle HS, Dickson EE, Luby JJ, Hokanson SC. Collection, maintenance, characterization and utilization of wild apples of central Asia. Horticultural Rev 2003; 29: 1-62.

22 Bai Y, Dougherty L, Li M, Fazio G, Cheng L, Xu K. A natural mutation-led truncation in one of the two aluminum-activated malate transporter-like genes at the $\mathrm{Ma}$ locus is associated with low fruit acidity in apple. Mol Genet Genomics 2012; 287: 663-678.

23 Wang A, Xu K. Characterization of two orthologs of REVERSION-TO-ETHYLENE SENSITIVITY1 in apple. J Mol Biol Res 2012; 2: 24-41.

24 Blanpied GD, Silsby KJ. Predicting harvest date windows for apples. In: Information Bulletin 221: Cornell Cooperative Extension. Cornell University: Ithaca, NY, USA, 1992.

25 Neff MM, Turk E, Kalishman M. Web-based primer design for single nucleotide polymorphism analysis. Trends Genet 2002; 18: 613-615.

26 Marić S, Lukić M. Allelic polymorphism and inheritance of MdACS1 and MdACO1 genes in apple (Malus $\times$ domestica Borkh.). Plant Breed 2014; 133: 108-114.

27 Nybom H, Ahmadi-Afzadi M, Sehic J, Hertog M. DNA marker-assisted evaluation of fruit firmness at harvest and post-harvest fruit softening in a diverse apple germplasm. Tree Genet Genomes 2013; 9: 279-290.

28 Kondo S, Tomiyama H, Kittikorn M, Okawa K, Ohara H, Yokoyama M et al. Ethylene production and 1-aminocyclopropane-1-carboxylate (ACC) synthase and ACC oxidase gene expression in apple fruit are affected by 9,10-ketol-octadecadienoic acid (KODA). Postharvest Biol Technol 2012; 72: 20-26.

29 Bulens I, Van de Poel B, Hertog MLATM, Cristescu SM, Harren FJM, De Proft MP et al. Dynamic changes of the ethylene biosynthesis in 'Jonagold' apple. Physiol Plantarum 2014; 150: 161-173.

30 Varanasi V, Shin S, Mattheis J, Rudell D, Zhu Y. Expression profiles of the MdACS3 gene suggest a function as an accelerator of apple (Malus $\times$ domestica) fruit ripening. Postharvest Biol Technol 2011; 62: 141-148.

31 Busatto N, Farneti B, Tadiello A, Velasco R, Costa G, Costa F. Candidate gene expression profiling reveals a time specific activation among different harvesting dates in 'Golden Delicious' and 'Fuji' apple cultivars. Euphytica 2016; 208: 401-413.

32 Kamath OC, Kushad MM, Barden JA. Postharvest quality of 'Virginia Gold' apple fruit. Fruit Var J 1992; 46: 87-92.

33 Pech JC, Bouzayen M, Latché A. Climacteric fruit ripening: ethylene-dependent and independent regulation of ripening pathways in melon fruit. Plant Sci 2008; 175: $114-120$.

34 Bemer M, Karlova R, Ballester AR, Tikunov YM, Bovy AG, Wolters-Arts $M$ et al. The tomato FRUITFULL homologs TDR4/FUL1 and MBP7/FUL2 regulate ethyleneindependent aspects of fruit ripening. Plant Cell 2012; 24: 4437-4451.

35 King RC, Mulligan P, Stansfield W. A Dictionary of Genetics, 8th edn. Oxford University Press: New York, 2013.

36 Rédei GP. Encyclopedia of Genetics, Genomics, Proteomics, and Informatics, 3rd edn. Springer Science \& Business Media: Dordrecht, The Netherlands, 2008.

This work is licensed under a Creative Commons Attribution 4.0 International License. The images or other third party material in this article are included in the article's Creative Commons license, unless indicated otherwise in the credit line; if the material is not included under the Creative Commons license, users will need to obtain permission from the license holder to reproduce the material. To view a copy of this license, visit http://creativecommons.org/licenses/ by/4.0/

Supplementary Information for this article can be found on the Horticulture Research website (http://www.nature.com/hortres) 